\section{Doppler ultrasonography of the lower extremity arteries: anatomy and scanning guidelines}

\author{
Ji Young Hwang \\ Department of Radiology, Ewha Womans University School of Medicine, Seoul, Korea
}

Doppler ultrasonography of the lower extremity arteries is a valuable technique, although it is less frequently indicated for peripheral arterial disease than for deep vein thrombosis or varicose veins. Ultrasonography can diagnose stenosis through the direct visualization of plaques and through the analysis of the Doppler waveforms in stenotic and poststenotic arteries. To perform Doppler ultrasonography of the lower extremity arteries, the operator should be familiar with the arterial anatomy of the lower extremities, basic scanning techniques, and the parameters used in color and pulsed-wave Doppler ultrasonography.

Keywords: Arteries; Lower extremity; Ultrasonography, Doppler, color; Ultrasonography, Doppler, pulsed; Peripheral arterial disease

\section{Introduction}

Imaging modalities for evaluating peripheral arterial disease in the lower extremities include computed tomography (CT) angiography, conventional angiography, and Doppler ultrasonography (US). Three-dimensional CT angiography provides information about atherosclerotic calcifications and the extent of stenosis or occlusion of the arteries. CT angiography has some advantages, such as a shorter examination time, the ability to evaluate the iliac artery, and the fact that it is less affected by the operator's experience. Conventional angiography is used for vascular interventions such as angioplasty or stent application, as well as in the diagnosis of peripheral arterial disease. Doppler US is the only noninvasive technique that does not require contrast enhancement, preparation of the patient before the study, or radiation exposure [1,2]. Doppler US is a good method for screening and follow-up, as well as for the definitive diagnosis of peripheral arterial disease [3-7]. Color Doppler US can easily identify arteries by finding round objects with regular pulsation and can be used to detect stenotic or occluded segments $[4,8]$. Pulsed-wave Doppler US can show the exact flow velocity of each arterial segment and determine the degree of severity of the stenosis based on an analysis of the pulsed-wave Doppler spectral waveform [9].

Knowledge of the ultrasonographic anatomy of the lower extremity arteries and the corresponding anatomical landmarks is essential for performing Doppler US. In this article, we review the basic scanning techniques of color and pulsed-wave Doppler US for the lower extremity arteries and the spectral analysis of normal and stenotic arteries on pulsed-wave Doppler US.

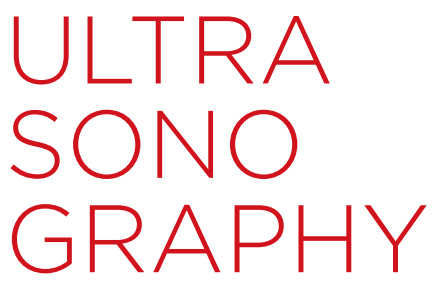

\section{REVIEW ARTICLE}

https://doi.org/10.14366/usg. 16054 pISSN: 2288-5919 • elSSN: 2288-5943 Ultrasonography 2017;36:111-119

Received: December 30, 2016 Revised: January 17, 2017 Accepted: January 18, 2017

Correspondence to: Ji Young Hwang, MD, Department of Radiology, Ewha Womans University School of Medicine, 1071 Anyangcheon-ro, Yangcheon-gu, Seoul 07985, Korea

Tel. +82-2-2650-5687

Fax. +82-2-2650-5302

E-mail: mshjy@ewha.ac.kr

This is an Open Access article distributed under the terms of the Creative Commons Attribution NonCommercial License (http://creativecommons.org/ licenses/by-nc/3.0/) which permits unrestricted noncommercial use, distribution, and reproduction in any medium, provided the original work is properly cited.

Copyright (C) 2017 Korean Society of Ultrasound in Medicine (KSUM)

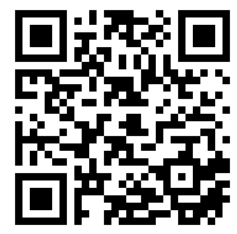

How to cite this article:

Hwang JY. Doppler ultrasonography of the lower extremity arteries: anatomy and scanning guidelines. Ultrasonography. 2017 Apr;36(2):111-119. 


\section{Anatomy of the Lower Extremity Arteries on CT Angiography}

Each lower extremity artery is visible with an accompanying vein, extending from the iliac artery to the popliteal artery. The anterior tibial artery, the posterior tibial artery, and the peroneal artery are seen with two homonymous veins. The overall anatomy of the arteries in the lower extremities is shown on CT angiography in Fig. 1.

The common iliac artery splits into the internal iliac artery and the external iliac artery in the pelvic cavity. The external iliac artery is continuous with the common femoral artery (Fig. 1A). The inguinal ligament is a landmark for the junction of the external iliac artery and common femoral artery. The inguinal ligament is located more proximally than the inguinal crease. The common femoral artery is a short segment, generally about $4 \mathrm{~cm}$ long, and bifurcates into the superficial femoral artery medially and the deep femoral artery laterally [10]. The superficial femoral artery descends without prominent branching between the quadratrus and adductor muscle groups in the anteromedial thigh. In the distal thigh, the superficial femoral artery enters the adductor canal. On leaving the adductor hiatus, the name of the artery becomes the popliteal artery in the popliteal fossa and ends by bifurcating into the anterior tibial artery and the tibioperoneal trunk in the posterior aspect of the proximal calf [11].

Below the knee, the anterior tibial artery passes from the posterior to the anterior, and then descends along the interosseous membrane behind the anterior tibialis muscle and the extensor muscles in the anterolateral leg. The tibioperoneal trunk divides into the posterior tibial artery medially and the peroneal artery laterally (Fig. 1B). The posterior tibial artery runs along the intermuscular space between

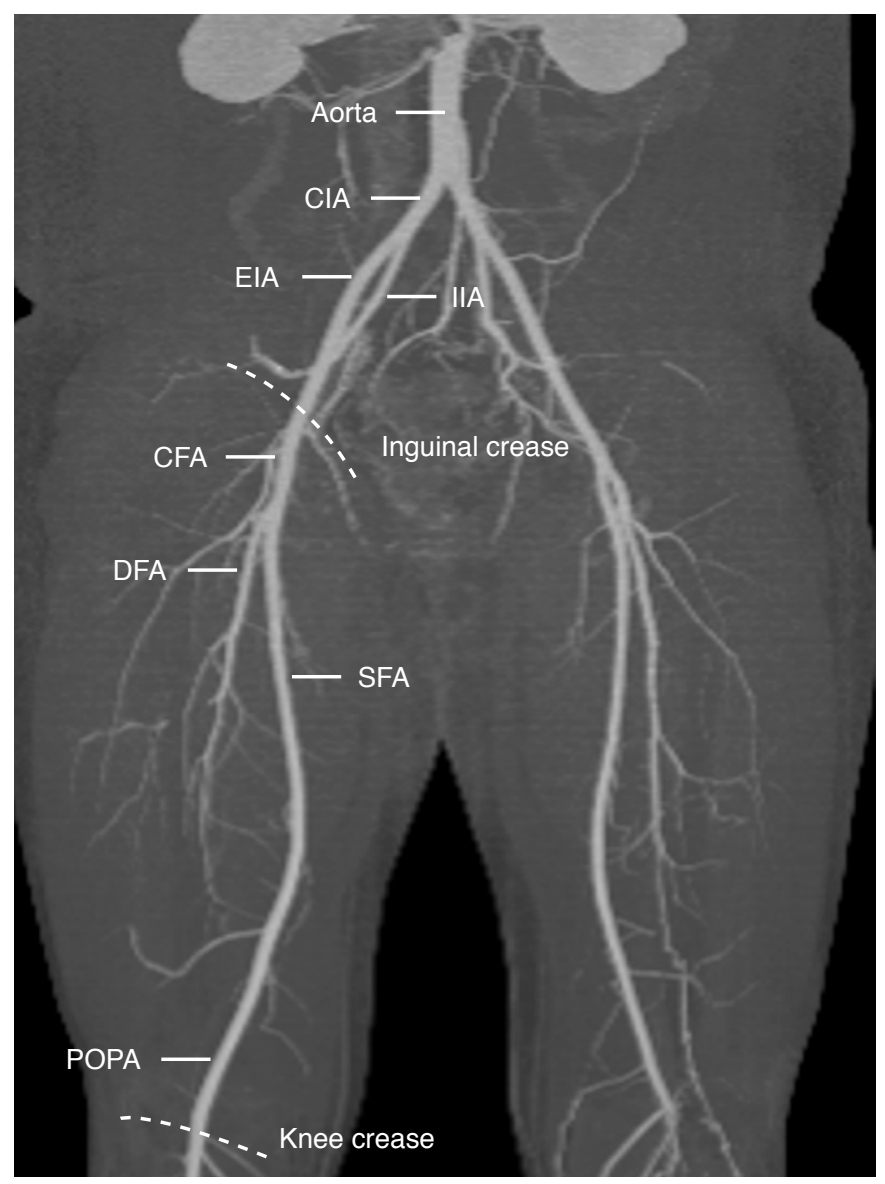

A

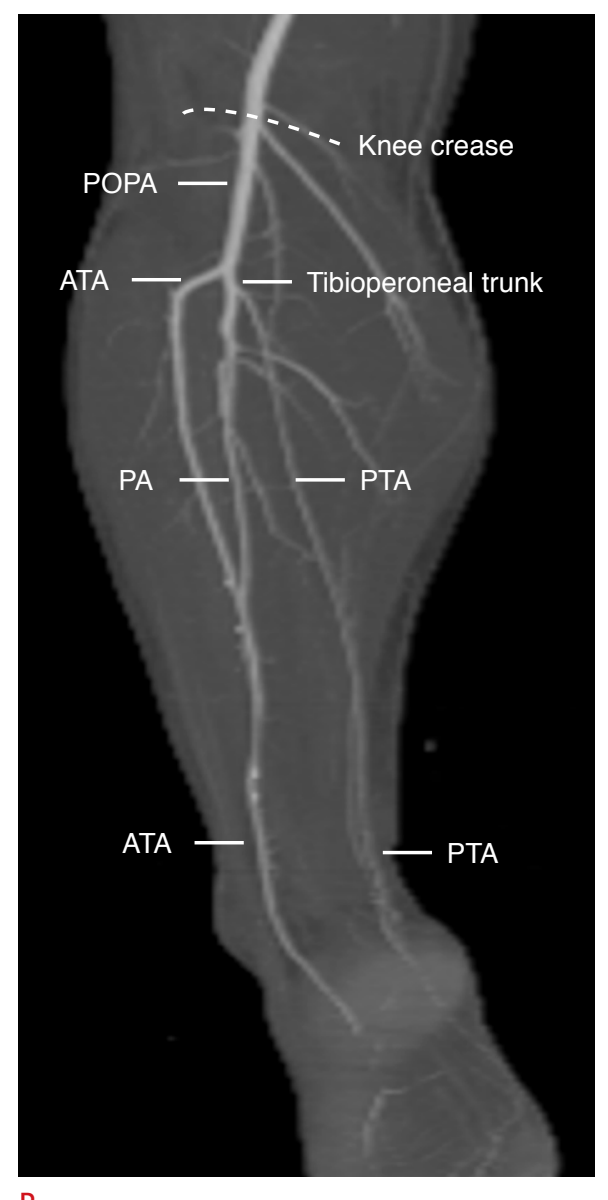

B

Fig. 1. The anatomy of the lower extremity arteries on computed tomography (CT) angiography.

A. On coronal maximal intensity projection (MIP) CT image above the knee, the external iliac artery (EIA) is continuous with the common femoral artery (CFA) which bifurcates into the superficial femoral artery (SFA) and deep femoral artery (DFA). The SFA is continuous with the popliteal artery (POPA). B. On coronal MIP CT image below the knee, the POPA splits anterior tibial artery (ATA) and tibioperoneal trunk which bifurcates into posterior tibial artery (PTA) and peroneal artery (PA). CIA, common iliac artery; IIA, internal iliac artery. 
the posterior tibialis muscle and the soleus muscles. The peroneal artery extends down between the posterior tibialis muscle and the flexor hallucis longus muscle.

In the ankle and foot region, the anterior tibial artery continues into the dorsalis pedis artery distal to the extensor retinaculum [11]. The dorsalis pedis artery forms the arcuate artery at the metatarsal base and gives rise to the dorsal metatarsal artery. The posterior tibial artery passes behind the medial malleolus of the tibia and bifurcates, forming the medial and lateral plantar arteries. The deep plantar arch from the medial and lateral plantar arteries gives rise to the plantar metatarsal and digital arteries of the foot [11].

\section{US Anatomy of the Lower Extremity Arteries}

Arteries can be differentiated from veins on US by several characteristics. First, arteries are round in transverse images, while veins are somewhat oval. Second, arteries are smaller than veins. Third, arteries have visible walls and sometimes have calcified plaques on the walls. Fourth, when the vessels are compressed by the transducer, arteries are partially compressed, while veins are completely collapsed [12].

Doppler US of the lower extremity begins at the inguinal crease by putting a transducer on the common femoral artery in the transverse plane with the patient in the supine position (Fig. 2). The common femoral artery is seen lateral to the femoral vein, which is drained from the greater saphenous vein anteromedially at the inguinal area (Fig. 3A). Just below the inguinal crease, the superficial femoral artery and the deep femoral artery are present alongside the femoral vein, showing a shape reminiscent of Mickey Mouse's face on a transverse scan (Fig. 3B). The common femoral artery, the bifurcated superficial femoral artery and deep femoral artery are seen in a fallen-Y configuration in a longitudinal scan (Fig. 2). From the proximal to distal thigh, scanning is performed by moving a transducer distally along the superficial femoral artery deep to the sartorius muscle. The superficial femoral artery goes together with the femoral vein (Fig. 2).

The popliteal artery is evaluated from the knee crease level in the transverse plane and then traced proximally up to the adductor
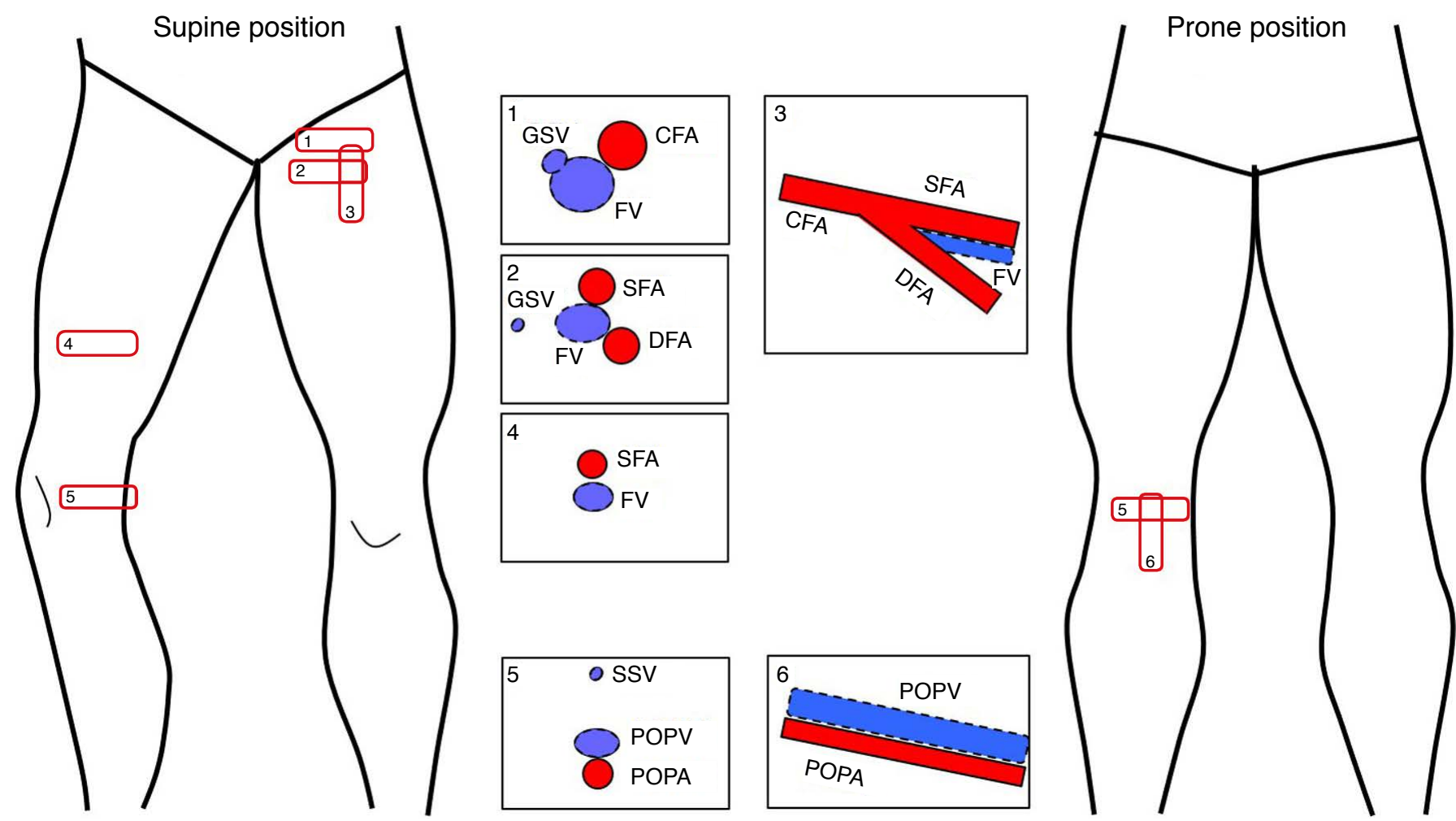

Fig. 2. The steps of color Doppler ultrasonography (US) for the lower extremities above the knee, with the patient's position indicated. The red rectangular boxes are the essential scanning sites and planes for the femoral arteries and the popliteal artery. The numbers within the boxes represent the general steps of scanning. The schema in the box demonstrates the typical US features of arteries and veins at each scanning site. GSV, greater saphenous vein; FV, femoral vein; CFA, common femoral artery; SFA, superficial femoral artery; DFA, deep femoral artery; SSV, small saphenous vein; POPV, popliteal vein; POPA, popliteal artery. 


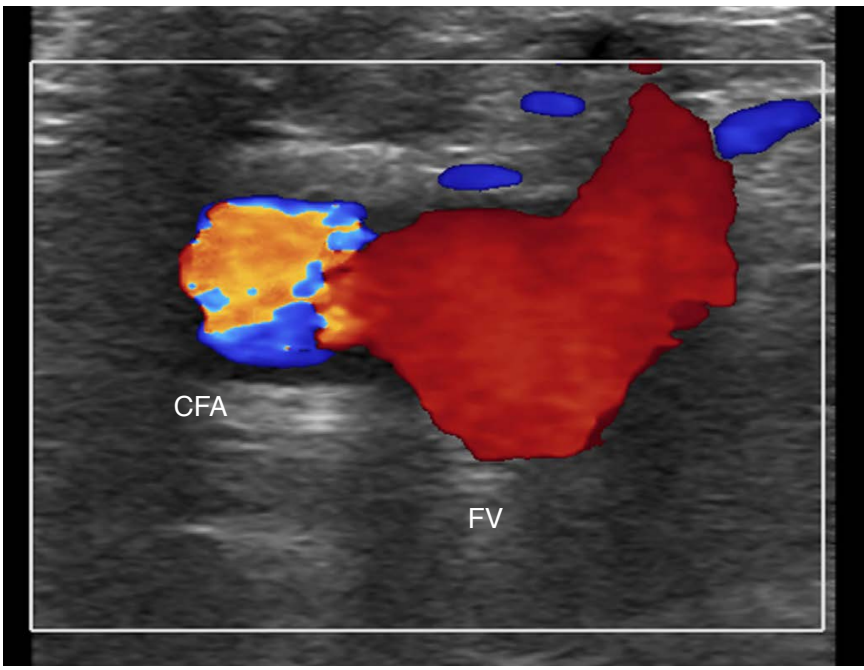

A

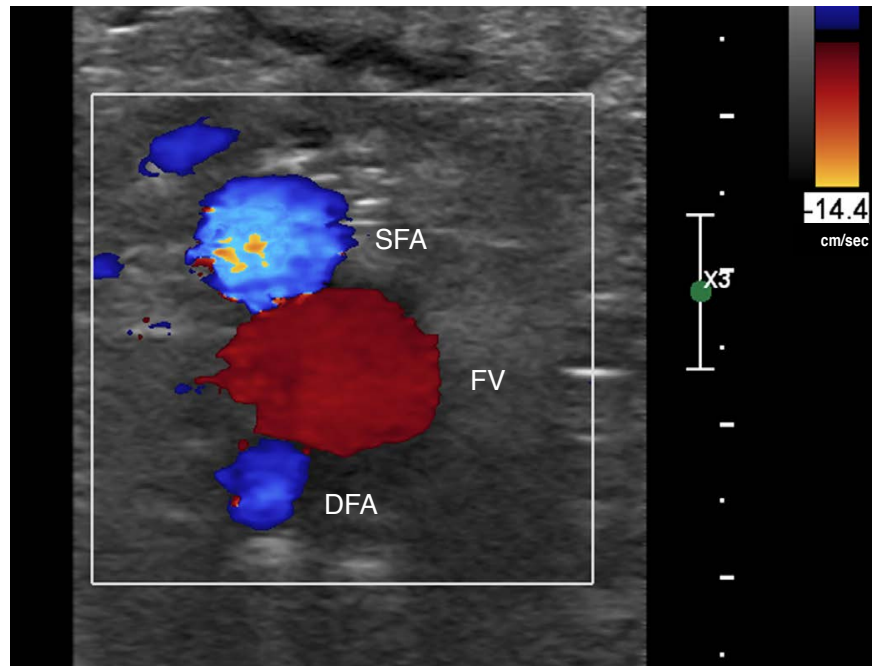

B

Fig. 3. Normal color Doppler ultrasonography of the femoral arteries in the inguinal area.

A. The common femoral artery (CFA) is lateral to the femoral vein (FV) on a transverse scan at the inguinal crease. Note that the size of the color box is as small as possible. B. The superficial femoral artery (SFA) and the deep femoral artery (DFA) make a shape like Mickey Mouse's ears, and the FV forms Mickey Mouse's face.

canal at the supracondylar level of the femur (Fig. 2). The popliteal artery is seen in the central portion of popliteal fossa between the medial and lateral heads of the gastrocnemius muscles. The evaluation of the posterior tibial artery can be started from its origins at the tibioperoneal trunk, if scanning distally, or from the ankle behind the medial malleolus, if scanning proximally (Fig. 4). The peroneal artery is scanned along the lateral side of the posterior calf and is visualized alongside the fibular bone (Fig. 4).

The evaluation of the anterior tibial artery can be started from the ankle anterior to the talus neck and continued proximally or started from the proximal anterolateral leg between the tibia and the fibula and continued distally (Fig. 4). The transducer is traced from the anterior ankle to the dorsal foot to evaluate the dorsalis pedis artery, continuing to the first dorsal metatarsal artery between the first and second metatarsal bones (Fig. 4).

\section{US Technique}

\section{Transducer and Patient Position}

A linear transducer with a variable ultrasound frequency of 9-15 $\mathrm{MHz}$ is generally used, but a convex transducer with a lower frequency can be selected for the evaluation of iliac arteries in the pelvic cavity [13]. The transducer is placed over an artery for transverse scanning, and then is rotated $90^{\circ}$ for longitudinal scanning. The artery should be scanned on a longitudinal plane as long as possible. The operator should rotate or move the transducer delicately to maintain visualization of the artery. Pulsed-wave Doppler US is performed in the longitudinal plane.

The examination is usually performed with the patient placed in the supine position. The patient's hip is generally abducted and externally rotated, and the knee is flexed like frog legs in order to easily approach the popliteal artery in the popliteal fossa and the posterior tibial artery in the medial calf (Fig. 2). The left lateral decubitus position or the prone position are the alternatives for evaluating the popliteal artery, the posterior tibial artery, and the peroneal artery (Fig. 4). The anterior tibial artery and dorsalis pedis artery are scanned in the supine position (Fig. 4).

\section{Parameters and Optimization of Doppler US}

The operator should be aware of both color and pulsed-wave Doppler parameters and how to adjust these parameters to obtain an optimal Doppler image.

Among these parameters, the color box, color gain, color velocity scale, and inversion are frequently used during color Doppler US scanning. The color box is a square area within the grayscale sonogram in which all color Doppler information is displayed (Fig. 3). The size and location of the box are adjustable, and the image resolution and quality are affected by the box size and depth [14]. The box should be made as small and placed as superficially as possible, thereby maximizing the frame rate. The color box should be tilted using the "steer" button according to the arterial axis during longitudinal scanning (Fig. 5). Color gain refers to the amplification 


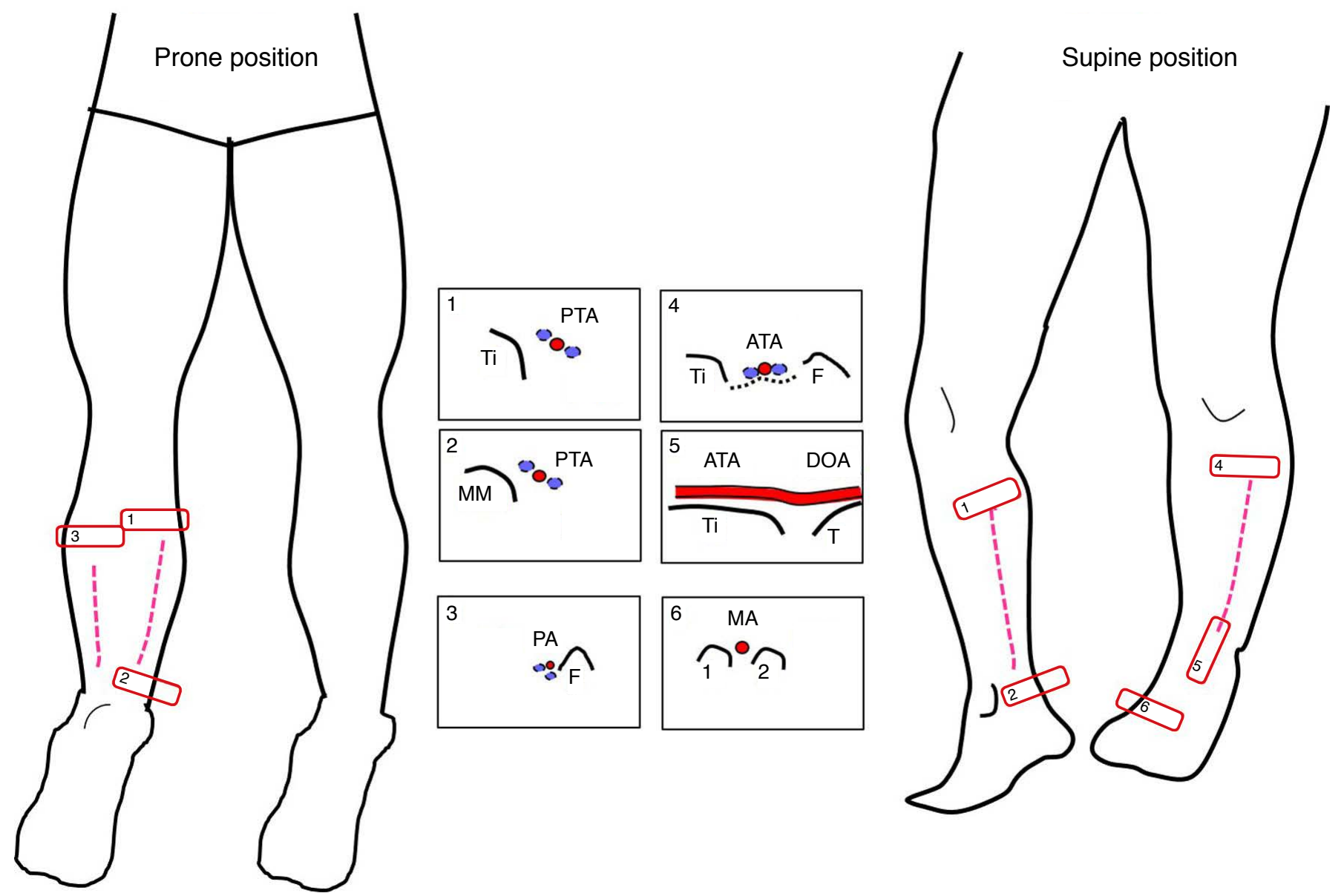

Fig. 4. The steps of color Doppler ultrasonography for the lower extremities below the knee, with the patient's position indicated. The posterior tibial artery (PTA) is seen along the tibia (Ti) at the medial side of the posterior calf (box 1) and behind the medial malleolus (MM) of the ankle (box 2). The peroneal artery (PA) is depicted alongside the fibula (F) on the lateral side of posterior calf in the prone position (box 3). The anterior tibial artery (ATA) is detected over interosseous memberane (black dashed line) between the tibia (Ti) and the fibula (F) at the anterolateral side of the calf (box 4). At the ankle level, the ATA is seen anterior to the tibia plafond (Ti) and the talus (T) (box 5) and continues to the dorsalis pedis artery (DOA) distal to the ankle and metatarsal artery (MA) between the metatarsal bones (box 6).

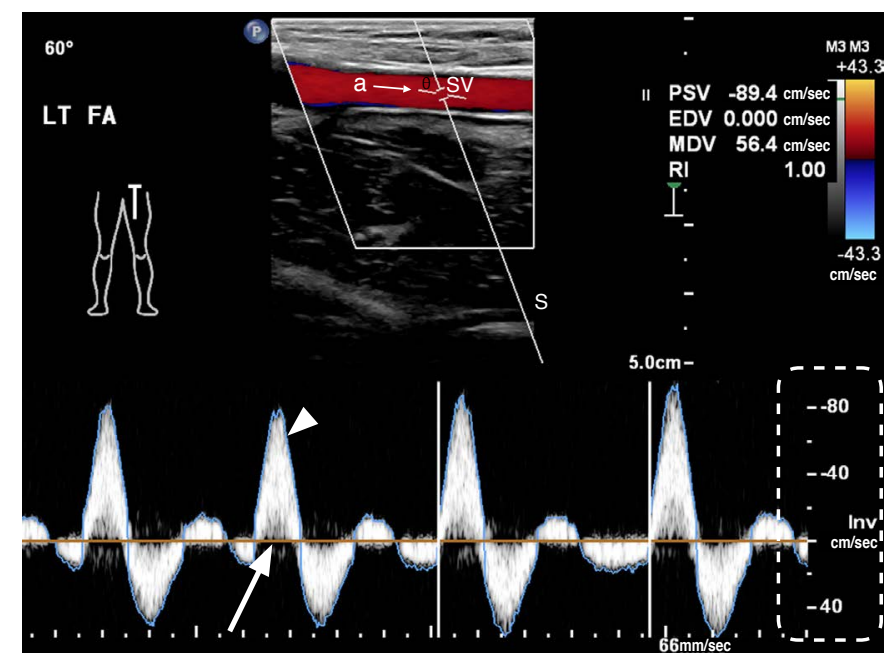

Fig. 5. Color and pulsed-wave Doppler sonograms of normal lower extremity arteries with parameters. Top: In a color image of a pulsed-wave Doppler sonogram, the color box is tilted to be parallel to the arterial axis using the "steer" key. Doppler angle $(\theta)$ is $60^{\circ}$ in this case and is formed by the Doppler line of sight (S) and the axis of arterial flow (a). SV, sample volume; LT FA, left femoral artery; PSV, peak systolic velocity; EDV, end-diastolic velocity; MDV, minimum diastolic velocity; RI, resistivity index. RI=(PSV-EDV)/PSV. Bottom: On the Doppler spectrum, time (seconds) is represented on the $x$-axis. Blood flow velocity $(\mathrm{cm} / \mathrm{sec})$ is shown on the $y$-axis (dashed line). Flow direction relative to the transducer is shown in relation to the spectrum baseline (arrow). The "high-Q" is the blue outline of the Doppler spectrum (arrowhead). 
of flow data to improve the depiction of flow [14]. Color gain (the "gain" button on the US machine) should be set as high as possible without displaying background color noise. The color velocity is the range of flow velocities that are depicted in color Doppler US [14]. If the velocity scale (the "scale" button on the US machine) setting is lower than the flow velocity of the artery, aliasing artifacts will be present. An operator can detect the color flow within an arterial lumen by increasing the gain or decreasing the scale. Color flow artifacts outside an artery should be removed by decreasing the gain. A homogeneous color of arterial flow can be obtained by increasing the scale. Flow toward the transducer typically appears red on color Doppler sonograms when a red color appears above the baseline on the color bar. Inversion can electronically invert the direction of flow, which may complicate the interpretation of the flow direction. Therefore, the flow direction should be interpreted based on the setting of the color bar. The wall filter (WF) eliminate the low frequency noise that may arise from vessel wall motion below an operator-defined frequency threshold $[9,14]$. WF settings are usually preset by manufacturer (Fig. 6).

It is important to understand the meaning of the parameters of pulsed-wave Doppler US and how to adjust them. The sample volume cursor is composed of parallel lines on both sides of the arterial axis line. The sample volume should be placed within an arterial lumen, and the range of the sample volume size is generally from one-third to one-half of the luminal diameter [15]. The Doppler angle is formed by the Doppler line and the axis of arterial flow

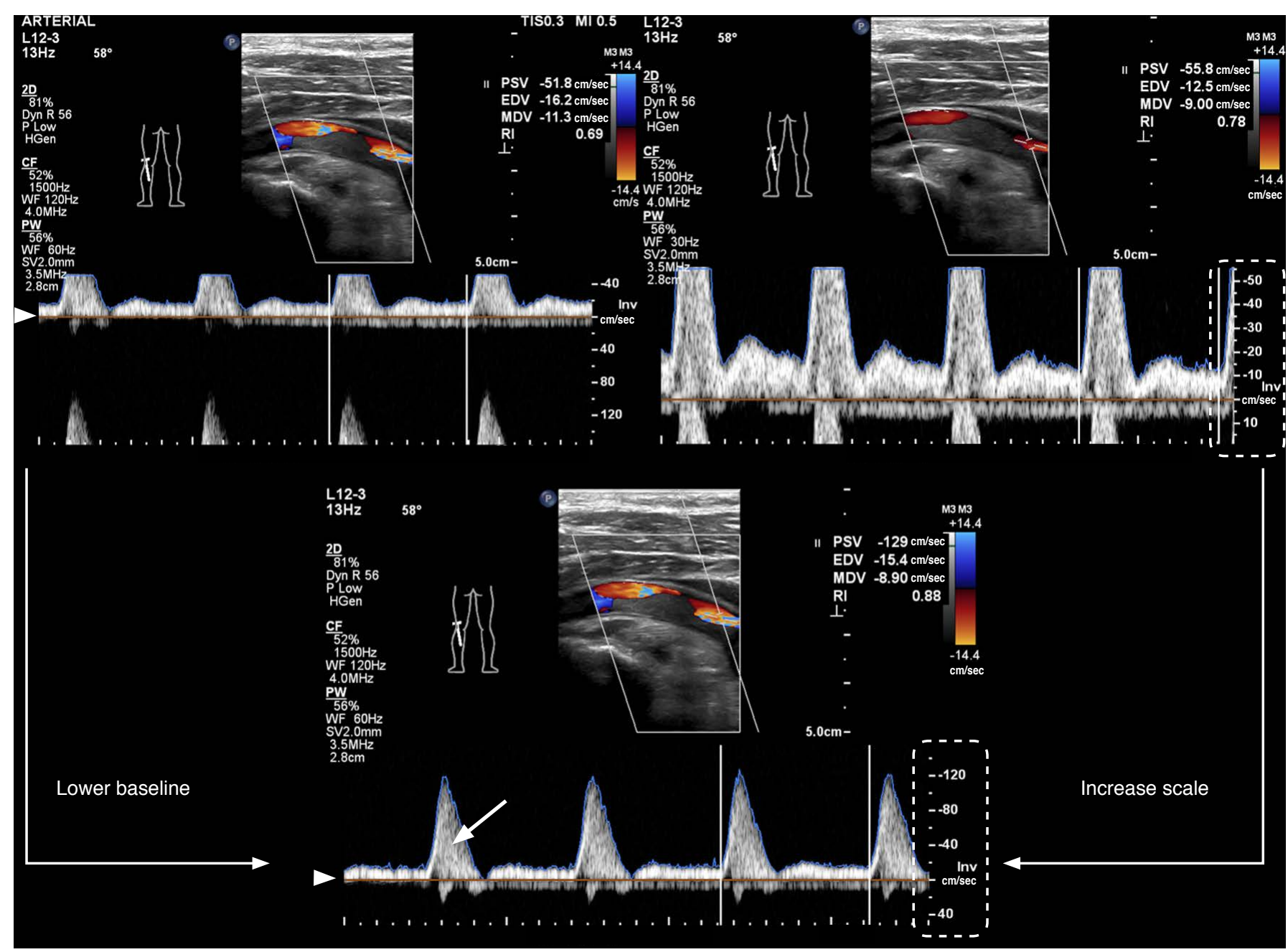

Fig. 6. Adjustment of pulsed-wave Doppler ultrasonography in a stenotic arterial segment. The aliasing artifact on the Doppler spectrum can be adjusted by lowering the baseline (arrowhead) and increasing the scale. Note the spectral broadening (arrow) in the Doppler spectrum due to stenosis of the artery. Parameters for color flow (CF) and pulsed-wave (PW) Doppler are peak systolic velocity (PSV) $129 \mathrm{~cm} / \mathrm{sec}$, end diastolic velocity (EDV) $15.4 \mathrm{~cm} / \mathrm{sec}$, minimum diastolic velocity (MDV) $8.9 \mathrm{~cm} / \mathrm{sec}$, resistivity index (RI) 0.88 , and wall filter (WF) $120 \mathrm{~Hz}$ in CF and $60 \mathrm{~Hz}$ in PW. SV, sample volume. 
and should be between $45^{\circ}$ and $60^{\circ}$ for optimal accuracy [9]. On Doppler US, the line in the center of the artery indicates of the axis of arterial flow. The nearly vertical line is the Doppler line of sight (Fig. 5). The Doppler spectrum is a graph showing the mixture of frequencies over a short period of time [9]. The Doppler frequency is defined as the difference between the received and transmitted frequencies when blood cells are moving. The key elements of the Doppler spectrum are the time and velocity scales [9]. On the Doppler spectrum, time (seconds) is represented on the $\mathrm{x}$-axis, and the velocity scale $(\mathrm{cm} / \mathrm{sec})$ is shown on the $y$-axis (Fig. 5). Flow direction relative to the transducer is shown in relation to the spectrum baseline. Flow toward the transducer is represented by positive velocity above the baseline (Fig. 5). The "high-Q" or peak velocity envelope is the blue outline surrounding the Doppler spectrum. Based on this envelope, peak systolic velocity (PSV), minimum diastolic velocity (MDV), end-diastolic velocity (EDV), and the resistivity index (RI) can be obtained numerically (Figs. 5, 6). PSV is the highest systolic velocity, MDV is the lowest diastolic velocity, and EDV is the highest end-diastolic velocity. The RI is one of popular measurements of the pulsatility representing peripheral flow resistance [9]. If an aliasing artifact is present in the Doppler spectrum, the baseline can be reduced or the scale increased in order to optimize the velocity range (Fig. 6). It can be automatically optimized by pressing the "i-scan" button.

\section{Doppler Spectrum of Normal Lower Extremity Arteries}

The Doppler waveform of the lower extremity arteries at rest is classified as a high pulsatility waveform and is characterized by a triphasic flow pattern [9]. Over the course of each heartbeat, a tall, narrow, and sharp systolic peak in the first phase is followed by early diastolic flow reversal in the second phase, and then by late diastolic forward flow in the third phase (Fig. 5). Diastolic flow reversal results from the high peripheral resistance of normal extremity arteries [9]. In normal extremity arteries, flow acceleration in systole is rapid, which means that the peak velocity is reached within a few hundredths of a second after ventricular contraction begins. Blood in the center of the artery moves faster than blood at the periphery, which is described as laminar flow [9]. When the flow is laminar, the blood cells are moving at a similar speed. These features of normal arteries produce a clear space, known as the spectral window, under the Doppler spectrum.

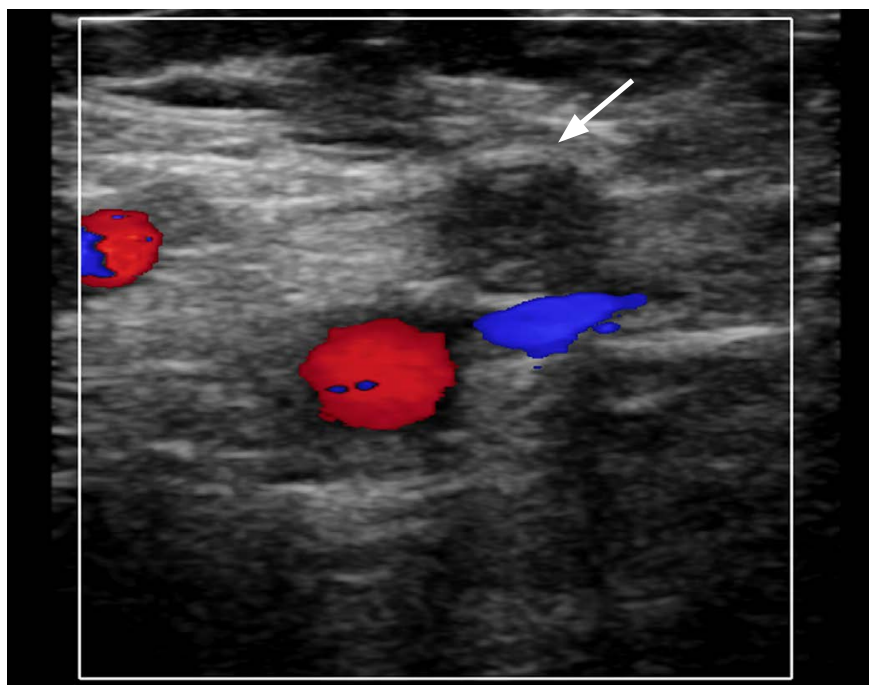

Fig. 7. A 56-year-old man with arterial occlusion. Color flow is absent in the superficial femoral artery (arrow) on color Doppler sonogram at the inguinal level, representing complete occlusion. The red vessel is the deep femoral artery and the blue vessel is the collapsed femoral vein.

\section{Abnormal US Findings}

Doppler US of the lower extremities can be performed by obtaining grayscale images before the color Doppler study; however, grayscale evaluations are sometimes optional and can be considered as a second step when a color Doppler study yields findings suspicious for stenosis or occlusion. On a grayscale image, the presence and the size of a plaque should be described, as well as whether the plaque is calcified or not. Plaque size can be measured in terms of height and length obtained using transverse and longitudinal scans, respectively [16]. Three-dimensional US has been recently used for measuring plaque volume, with good intraobserver and interobserver reproducibility [17].

On color Doppler US, if an occlusion is present in the artery, color flow is absent within the lumen (Fig. 7).

The Doppler spectrum at severely stenotic arterial segments and poststenotic or post-obstructive distal arterial segments is briefly reviewed below. The peak systolic velocity at stenotic segments increases until the diameter is reduced by $70 \%$, which corresponds to a $90 \%$ reduction in area [18]. The area of flow disturbance showing spectral broadening occurs within $2 \mathrm{~cm}$ beyond the area of stenosis due to loss of the laminar flow pattern (Fig. 6). Spectral broadening is prominent, with a diameter reduction of $20 \%$ $50 \%$. The lower extremity artery waveform may convert to a lowresistance form with low pulsatility after exercise or as a result of 
the occlusion of more proximal arteries [18]. If the waveform is monophasic, that means that the entire waveform is either above or below the Doppler spectrum baseline, depending on the orientation of the US transducer [9]. It is characterized by a "damped" pattern, which means that systolic flow acceleration is slowed, peak systolic velocity is reduced, and diastolic flow is increased [19]. This monophasic waveform is seen at the stenotic site and in the distal artery in cases of severe stenosis with a diameter reduction of more than $50 \%$.

\section{Conclusion}

Doppler US has been found to distinguish between stenosis with a diameter reduction greater than or less than 50\% (corresponding to an area reduction of $70 \%$ ) with a sensitivity of $77 \%-82 \%$ and a specificity of $92 \%-98 \%[18,20-24]$. Complete scanning of both lower extremities may require up to 2 hours depending on the operator's experience [18]. However, if the operator is familiar with the US anatomy of the lower extremity arteries and understands the parameters and Doppler waveforms of Doppler US, accurate diagnostic results can be produced with less scanning time.

ORCID: Ji Young Hwang: http://orcid.org/0000-0001-5848-3429

\section{Conflict of Interest}

No potential conflict of interest relevant to this article was reported.

\section{References}

1. Nzeh DA, Allan PL, McBride K, Gillespie I, Ruckley CV. Comparison of colour Doppler ultrasound and digital subtraction angiography in the diagnosis of lower limb arterial disease. Afr J Med Med Sci 1998:27:177-180.

2. Karacagil S, Lofberg AM, Granbo A, Lorelius LE, Bergqvist D. Value of duplex scanning in evaluation of crural and foot arteries in limbs with severe lower limb ischaemia: a prospective comparison with angiography. Eur J Vasc Endovasc Surg 1996;12:300-303.

3. Flanigan DP, Ballard JL, Robinson D, Galliano M, Blecker G, Harward TR. Duplex ultrasound of the superficial femoral artery is a better screening tool than ankle-brachial index to identify at risk patients with lower extremity atherosclerosis. J Vasc Surg 2008;47:789-792.

4. Sensier Y, Bell PR, London NJ. The ability of qualitative assessment of the common femoral Doppler waveform to screen for significant aortoiliac disease. Eur J Vasc Endovasc Surg 1998;15:357-364.

5. Gooding GA, Perez S, Rapp JH, Krupski WC. Lower-extremity vascular grafts placed for peripheral vascular disease: prospective evaluation with duplex Doppler sonography. Radiology 1991;180:379-386.

6. Sorrell K, Demasi R. Delayed vascular injury: the value of follow-up color flow duplex ultrasonography. J Vasc Technol 1996;20:93-98.

7. Mohler ER 3rd, Bundens W, Denenberg J, Medenilla E, Hiatt WR, Criqui MH. Progression of asymptomatic peripheral artery disease over 1 year. Vasc Med 2012;17:10-16.

8. Hussain ST. Blood flow measurements in lower limb arteries using duplex ultrasound. Ann R Coll Surg Engl 1997;79:323-330.

9. Zwiebel WJ, Pellerito JS. Basic concepts of Doppler frequency spectrum analysis and ultrasound blood flow imaging. In: Zwiebel WJ, Pellerito JS, eds. Introduction to vascular ultrasonography. 5th ed. Philadelphia, PA: Elsevier Saunders, 2004;61-89.

10. Keck GM, Zwiebel WJ. Arterial anatomy of the extremities. In: Zwiebel WJ, Pellerito JS, eds. Introduction to vascular ultrasonography. 5th ed. Philadelphia, PA: Elsevier Saunders, 2004;265-274.

11. Schuenke M, Schulte E, Schumacher U. Lower limb. In: Ross LM, Lamperti ED, eds. Atlas of anatomy. New York: Thieme, 2006;464465

12. Hatsukami TS, Primozich J, Zierler RE, Strandness DE Jr. Color Doppler characteristics in normal lower extremity arteries. Ultrasound Med Biol 1992:18:167-171.

13. Zierler RE. Doppler techniques for lower extremity arterial diagnosis. Herz 1989;14:126-133.

14. Kruskal JB, Newman PA, Sammons LG, Kane RA. Optimizing Doppler and color flow US: application to hepatic sonography. Radiographics 2004;24:657-675.

15. Knox RA, Phillips DJ, Breslau PJ, Lawrence R, Primozich J, Strandness DE Jr. Empirical findings relating sample volume size to diagnostic accuracy in pulsed Doppler cerebrovascular studies. J Clin Ultrasound 1982;10:227-232.

16. Lee W. General principles of carotid Doppler ultrasonography. Ultrasonography 2014;33:11-17.

17. Landry A, Spence JD, Fenster A. Measurement of carotid plaque volume by 3-dimensional ultrasound. Stroke 2004;35:864-869.

18. Zierler RE. Ultrasound assessment of lower extremity arteries. In: Zwiebel WJ, Pellerito JS, eds. Introduction to vascular ultrasonography. 5th ed. Philadelphia, PA: Elsevier Saunders, 2004;341-356.

19. Kotval PS. Doppler waveform parvus and tardus: a sign of proximal flow obstruction. J Ultrasound Med 1989;8:435-440.

20. Jager KA, Phillips DJ, Martin RL, Hanson C, Roederer GO, Langlois

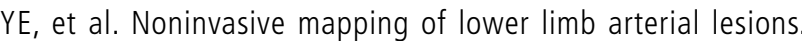
Ultrasound Med Biol 1985;11:515-521.

21. Kohler TR, Nance DR, Cramer MM, Vandenburghe N, Strandness DE Jr. Duplex scanning for diagnosis of aortoiliac and femoropopliteal disease: a prospective study. Circulation 1987;76:1074-1080.

22. Shaalan WE, French-Sherry E, Castilla M, Lozanski L, Bassiouny HS. Reliability of common femoral artery hemodynamics in assessing the severity of aortoiliac inflow disease. J Vasc Surg 2003;37:960969. 
23. Hussain ST, Smith RE, Wood RF, Bland M. Observer variability in volumetric blood flow measurements in leg arteries using duplex ultrasound. Ultrasound Med Biol 1996;22:287-291.
24. Moneta GL, Yeager RA, Antonovic R, Hall LD, Caster JD, Cummings $C A$, et al. Accuracy of lower extremity arterial duplex mapping. J Vasc Surg 1992;15:275-283. 